\title{
e-Phaïstos
}

e-Phaïstos

Revue d'histoire des techniques / Journal of the history

of technology

V-1 2016 | 2018

Ville et technique

\section{Pensée urbaine et administration technique de Macao (fin XIXe - début XXe siècle)}

Urban thinking and technique's administration of Macao (XIXth - XXth century)

\section{Monica Jorge Da Costa}

\section{OpenEdition}

Journals

Édition électronique

URL : http://journals.openedition.org/ephaistos/1121

DOI : 10.4000/ephaistos. 1121

ISSN : 2552-0741

Éditeur

IHMC - Institut d'histoire moderne et contemporaine (UMR 8066)

Référence électronique

Monica Jorge Da Costa, «Pensée urbaine et administration technique de Macao (fin XIXe - début XXe siècle) », e-Phaïstos [En ligne], V-1 2016 | 2018, mis en ligne le 18 janvier 2018, consulté le 30 avril 2019. URL : http://journals.openedition.org/ephaistos/1121 ; DOI : 10.4000/ephaistos. 1121

Ce document a été généré automatiquement le 30 avril 2019

Tous droits réservés 


\title{
Pensée urbaine et administration technique de Macao (fin XIXe - début XXe siècle)
}

Urban thinking and technique's administration of Macao (XIXth - XXth century)

\author{
Monica Jorge Da Costa
}

La ville est dans l'espace, toujours. Mais voici qu'au XIX

siècle, on lui impose d'être un espace réglé, aux cheminements droits, aux édifices dégagés, à l'architecture harmonieuse. L'espace embelli, en quelque sorte.

Maurice Agulhon ${ }^{1}$.

Comment réaliser une lecture urbaine d'une ville coloniale telle que Macao ? Fruit d'une histoire particulièrement riche, cette ville voit le jour avec l'apparition sur les côtes chinoises des premières caravelles portugaises et n'aura de cesse d'évoluer

. De ces zones de pêche et de marécages soumis aux intempéries et aux razzias de pirates naîtra bientôt le premier véritable comptoir occidental en Chine, administré par les Portugais, qui obtiennent ainsi la mainmise sur les droits d'entrée des occidentaux dans ce pays. Zone portuaire située au Sud-Est sur l'estuaire de la rivière des Perles, Macao a une position géographique privilégiée lui permettant alors d'atteindre la ville de Canton et ses marchés par voie fluviale. Pourtant, l'installation des Portugais n'est considérée que comme temporaire par les autorités chinoises du milieu du XVI

${ }^{e}$ au milieu du XIXe 
siècle. L'évolution de la ville et de ces constructions est donc, pendant plusieurs siècles, pensée comme provisoire. Seules les attaques des navires hollandais en 1622 et 1626 permettront la construction de la première véritable muraille de protection de la ville en pierre, en 1632. À partir de ce moment-là et jusqu'en 1842, la ville de Macao sera délimitée par son enceinte et aura une urbanisation typiquement médiévale : construction pérenne des édifices publics et religieux, puis construction d'habitations autour de ces monuments, noyaux urbains dans la ville

. De fait, l'émergence d'une pensée urbaine de la ville par les autorités portugaises sera concomitante à la prise de Hong-Kong par les Anglais. Ces derniers, obtenant les mêmes prérogatives que les Portugais, réussissent en peu de temps à supplanter Macao économiquement grâce à leur nouveau port et à des conditions géographiques plus favorables pour le transit d'un grand nombre de navires marchands. C'est donc dans ce contexte particulier de ville entre deux cultures et soumise à la concurrence de la colonie britannique voisine que naissent les premiers plans urbains de Macao.

siècle est un siècle charnière pour l'urbanisme en Europe et dans les colonies occidentales. Macao, colonie portugaise, n'échappe pas à l'arrivée des théories hygiénistes et à la volonté de rendre la ville saine. Toutefois, à la différence de nombreuses villes européennes, c'est à ce moment-là que naît véritablement la première trame urbaine de la ville organisée, et que naît la première administration apte à mettre en place une politique urbaine adaptée. L'étude des premiers plans urbains et des premiers projets nous permet de comprendre le renouvellement de la manière de penser la ville de Macao par ses dirigeants politiques locaux et métropolitains, mais aussi d'étudier en profondeur les mutations sociales, économiques et culturelles qui y surviennent. Si la ville de Macao est aujourd'hui méconnaissable dans son architecture et sa population, les divisions et les réhabilitations du XIX

siècle perdurent dans le temps par le maintien d'activités et d'espaces définis par les politiques de l'époque. Intéressons-nous donc à cette période de l'histoire de Macao qui court de la prise de Hong-Kong par les Anglais à la Seconde Guerre Mondiale et étudions comment la mise en place d'une administration technique urbaine et la mainmise de techniciens

sur les planifications ont permis un remodelage de la ville dans sa construction comme dans ses représentations. Nous nous intéresserons dans un premier temps à la mise en place de cette administration technique et au contexte historique du milieu du XIX siècle. Nous nous focaliserons ensuite sur les principaux plans urbains, leur application et leur ancrage dans la ville aujourd'hui.

Déclin économique d'une ville privilégiée 
La ville portuaire a été fondée en 1553 par les Portugais et a bénéficié dès le départ de prérogatives particulières puisqu'elle était la seule à accueillir des étrangers Seul comptoir de Chine, elle a permis aux Portugais de s'enrichir grâce aux droits de douane, et aux autorités chinoises de contrôler les allées et venues des occidentaux sur leur territoire. En effet, si la ville possédait son propre gouvernement local (le

Leal Senado

) et un gouverneur représentant l'autorité de la métropole, elle était aussi tenue de rendre des comptes au Grand Mandarin, représentant du gouverneur de la province du Guangdong.

L'arrivée des Anglais change la géopolitique du sud-est de la Chine. Ils s'imposent comme une nouvelle force et obtiennent avec le traité de Nankin de 1842, Hong-Kong et sa zone portuaire ainsi que des avantages douaniers et commerciaux non négligeables avec la Chine. Cet évènement politique a deux incidences principales sur Macao. Il y a d'abord la perte du statut d'unique ville de commerce des Européens avec la Chine. Pire, la ville et le port sont anciens et n'ont pas les moyens techniques de concurrencer le nouveau comptoir anglais mieux situé. L'économie décline et les différentes puissances occidentales marchandes se détournent de la ville, lui préférant sa voisine plus adaptée et aux conditions de vie meilleures. Cet évènement a aussi pour effet de voir le nouveau gouverneur portugais Ferrera do Amaral (1845-1880) réaffirmer l'autorité du Portugal sur

la ville et renvoyer le Grand Mandarin, représentant de l'autorité chinoise et ses fonctionnaires, en 1849. Il initiera alors une politique d'expansion de la ville. Macao se divisait jusqu'alors entre la ville portugaise enfermée dans ses murailles et les villages chinois situés sur son pourtour et dépendants de la ville mais sous autorité chinoise. Ferrera do Amaral repousse les limites de Macao jusqu'à l'actuelle frontière de cette Région Spéciale Administrée

${ }^{5}$ avec la Chine « Mainland $»^{6}$

. Il s'approprie les îles de Taipa et Coloane (1851 et 1864) et entreprend l'intégration de la population chinoise à la ville. Ce dernier point sera de ce moment une question récurrente dans les plans urbains du début du XX ${ }^{\mathrm{e}}$ siècle $\mathrm{e}^{7}$

\section{La « pré-urbanisation » à Macao : 1845 à 1881}

C'est sous le mandat de Ferrera do Amaral en effet que les premières initiatives urbaines

voient le jour à Macao

8

. Elles s'accompagnent d'un élargissement du territoire urbain à de nouveaux espaces jusque là inoccupés, de la disparition des portes de la ville et de la mise en place de voiries sur tout le territoire. Ces premières initiatives urbaines sont très peu connues cependant.

Elles eurent lieu dans les quartiers de la Horta da Mitra et de Volong au début du gouvernorat, sans donner toutefois les résultats escomptés. Dès le début du XX ${ }^{\mathrm{e}}$ siècle, ces quartiers étaient détruits, puis reconstruits. 
Les premiers véritables projets urbains sont l'œuvre de plusieurs hommes influents de Macao, qui, en mettant leur savoir en commun, permettront une première appréhension du territoire de Macao et une lecture de ses besoins en termes d'urbanisme. Le premier d'entre eux est le lieutenant Demétrio Cinatti qui s'intéresse particulièrement à la topographie, la cartographie et la climatologie. Lors de sa nomination au poste de capitaine des ports de Macao en 1878 et jusqu'à la fin de ses fonctions en 1885, il réalise des études sur la ville de Macao et dessine la première cartographie in situ

de la ville et de son port. La cartographie qu'il présente en 1881, malheureusement perdue aujourd'hui, permit non seulement d'avoir une vision générale de Macao, mais surtout « de signaler les ressources et potentiels de développement et d'expansion, mais aussi de détecter les foyers de dégradation et de maladie »

. La cartographie de Demétrio Cinatti mettait aussi en avant les caractéristiques géographiques, hydrographiques, maritimes et fluviales de la ville. Ce relevé attira l'attention des autorités sur l'état d'envasement du port extérieur de Macao et les convainquit de planifier des travaux de grande envergure afin de pouvoir maintenir le commerce maritime de la ville, sa première source de richesse

. Mais ce rapport eut aussi le mérite de mettre en lumière la nécessité de réorganiser la ville et de l'assainir. S'amorce alors une réflexion autour de la ville, de sa reconstruction et de l'amélioration des conditions de vie.

Macao, en cette fin de siècle, est une ville considérée comme sale et peu attrayante par les étrangers qui la visitent. Il existe plusieurs causes à cette image de la ville. La première est le manque d'hygiène et d'installations sanitaires, en particulier dans les quartiers chinois où les autorités ne sont que peu intervenues à la suite de l'intégration de ces populations, auparavant administrées par le Grand Mandarin. Voici la description par un voyageur portugais, Manuel de Castro Sampaio, du « Bazar chinois », un quartier édifié par les Chinois qui, au XVIIIe siècle, avaient progressivement investi le cœur de la ville de

11

ne sont pas hautes. Les plus élevées visuellement à Macao n'excèdent pas deux étages. [...] Aucune maison chinoise n'a suffisamment de ventilation et de lumière. Même les nouvelles, qui, en comparaison avec les anciennes, leur résulte une grande différence en leur faveur [revoir la traduction - ça ne veut rien dire], n'ont pour ainsi dire pas l'air et la lumière qui leur sont nécessaires. Toutes sont divisées et subdivisées. Les chins y vivent entassés avec des animaux domestiques [...]. Peu nombreux sont les compartiments qui ne renferment pas un grand nombre de personnes, mais toujours avec l'intention de conserver les sexes séparés. [...] Il y a toujours un grand manque de propreté et d'hygiène 12 . 》 
Les quartiers chinois sont insalubres et la ville connaît encore de nombreuses épidémies mortelles jusqu'au début du XX siècle : pestes, choléras, varioles et parfois méningites. La mise en place des premiers plans urbains avait donc pour objectif, outre l'aspect économique de la modernisation du port, de limiter la propagation des épidémies et d'instaurer des règles d'hygiènes dans la ville. Ainsi, celles-ci s'appliqueront essentiellement dans les quartiers chinois, la population la plus concernée, mais aussi la plus touchée par les épidémies mortelles ; les Portugais ayant plus facilement recours aux moyens médicaux à cette période

. Le premier décret, du 21 octobre 1863, instaure plusieurs règlements de politique urbaine. La ville s'engage à mettre en place une politique de nettoyage des rues et des équipements publics, de définir des matériaux de construction adaptés à l'humidité et à la lutte contre les incendies 13

, l'obligation de contrôler les habitations et leurs conditions d'hygiène, l'amélioration des politiques sanitaires par les services de santé de la ville et surtout l'interdiction de « la cohabitation entre les hommes et les animaux »

. C'est à la suite de ce décret que la première administration urbaine de la ville voit le jour.

\section{Administration urbaine et législations}

Cette administration urbaine nait de l'implication de plusieurs hommes influents de Macao qui s'attachèrent à trouver des solutions aux questions posées par Demétrio Cinatti. Deux figures principales marquent ce tournant : l'ingénieur José de Brito, qui instaura les premières réglementations sur le logement et la voirie, et le docteur Lúcio

Augusto Da Silva, qui apporta la participation du service public de la santé dans les discussions autour de l'urbanisation de la ville et des conditions de vie exigées pour celleci. Ainsi naît en 1883 la « Commission chargée d'étudier les améliorations de la ville de

» qui instaure tous les dispositifs de réglementations, mais aussi les premiers projets urbains.

Trois événements principaux ponctuent la première phase d'existence de cette commission locale. Le premier est l'émergence d'une pensée urbaine visant la ville dans son intégralité, avec des réflexions notamment sur la création d'égouts, la plantation de nombreux arbres, dite « révolution verte »

16

, la création de voiries reliant les pôles principaux de la ville et l'assainissement des fontaines d'eau potable. 
Si tous ces projets ne sont pas réalisés entre 1883 et 1900, on assiste néanmoins à une prise de conscience des réalités de la ville et de ses besoins à grande échelle. Le second événement est la mise en place des premières planifications de reconstruction de quartiers insalubres et d'un décret permettant l'expropriation des logements pour pouvoir détruire les quartiers contaminés. La réalisation des trois premiers projets urbains de réhabilitation de quartiers chinois a lieu entre 1885 et 1886 . Si tous ces projets ne sont pas réalisés entre 1883 et 1900, on assiste néanmoins à une prise de conscience des réalités de la ville et de ses besoins à grande échelle.

Les deux premiers projets urbains concernent les quartiers de Sákong et Sankiu ${ }^{17}$

et expérimentent l'alignement des constructions, l'éradication des déchets sur la voie publique mais surtout la création de canalisations pour l'évacuation des eaux usagées. Ces deux quartiers sont d'autant plus sensibles qu'ils se situent à proximité du port intérieur de Macao : l'un au niveau des docks, sur le canal Sákong et l'autre sur le canal de Sankiu allant jusqu'au centre de la ville. Ces deux quartiers étaient alors des déchetteries à ciel ouvert facilitant la transmission des maladies. Ce programme est lancé en 1885. Après l'expulsion des populations chinoises y habitant, ces deux quartiers sont détruits pour ensuite être reconstruits. Il est probable que ces populations aient dû se reloger en périphérie de la ville ou dans d'autres quartiers chinois. Le projet de la Horta da Mitra est lancé, bien que ce quartier ait déjà été concerné par une réhabilitation. En effet, si l'alignement des voiries est bien visible sur la cartographie de Demétrio Cinatti, les édifices n'auront tenu qu'une quinzaine d'années. La question des réglementations architecturales et urbanistiques (fondations des constructions, matériaux, hauteurs, etc.) se pose puisque Macao connaît des effondrements, mentionnés dans les archives.

La seconde phase de cette commission débute en 1904. Elle est marquée par la réalisation d'un plan général de réhabilitation de la ville, sur demande de la métropole. La commission ordonne alors la réhabilitation de nombreux autres quartiers chinois, l'expropriation et l'expulsion de la population dans le but d'assainir la ville, mais aussi et surtout, de relier les quartiers entre eux et d'agrandir l'espace habitable, répondant à la croissance du nombre de ses habitants. La liste des quartiers concernés s'allonge : LongTin-Chin, Seac-Lu-Tau, Tap-Seac, S.Miguel, Volung et S.Lázaro

${ }^{18}$. Le point final à cette programmation hygiéniste est la destruction duBazar Chinês et la création de l'avenue Almeida Ribeiro en 1915.

\section{Conséquences de ces politiques urbaines sur la ville}

Lors de la période de « pré-urbanisation » initiée par Ferrera do Amaral, la principale pensée urbaine de la ville était politique. Le but était d'occuper le territoire de Macao, les îles de Taipa et Coloane et d'assurer l'intégration des populations chinoises dans la ville. L'emprise territoriale traduisait les aspirations politiques d'un gouverneur qui cherchait 
Cette période de pré-urbanisation est suivie par l'émergence d'une réflexion sur la ville et ses difficultés autour de la cartographie de Demétrio Cinatti, par la création d'une commission chargée de l'organisation urbaine et par l'assainissement de la ville. Les réglementations mises en place et les projets réalisés furent à l'origine d'une transformation importante de la ville, mais aussi de l'expulsion des populations chinoises les plus pauvres. Le centre-ville devint alors très réglementé. L'étude détaillée de la construction de l'avenue Almeida Ribeiro, entre 1911 et 1945, permet de se rendre compte de la complexification des dossiers administratifs pour obtenir un permis de construire. Chaque construction devait être approuvée par une commission qui faisait appliquer les réglementations aux logements et commerces. Au fur et à mesure des réglementations, la commission instaura une nouvelle image de la ville, en interdisant la construction d'édifices trop différents de l'image souhaitée. Au final, la ville devint, au début du XX

siècle une vitrine du Portugal en Chine. Les plans urbains hygiénistes, destinés à contrôler la population, à en améliorer les conditions de vie, assurèrent en complément la pérennité des constructions. Ils contribuèrent à définir une nouvelle image de la ville. Aujourd'hui encore, ce sont ces façades que l'on regarde/contemple au cœur de la ville comme étant « l'architecture portugaise de Macao », inscrite depuis 2005 sur la liste

UNESCO du patrimoine de l'humanité.

C'est aussi pendant cette période de 1880 à 1920 que Macao acquière sa réputation de ville « verte » due aux politiques urbaines de plantation d'arbres, destinées à modifier la vision d'une ville dépourvue de végétation, entourée par des monts arides

. Les voiries larges permettent le transport de plusieurs carrioles à chevaux et l'éclairage au gaz apparait. Ces éléments participent à la construction d'une nouvelle image de Macao, touristique et attractive pour les commerçants et les négociants de Hong-Kong. La planification urbaine devient le vecteur de nouvelles politiques, en particulier économiques. La ville passe du statut de ville portuaire marchande à celui de ville touristique. Elle tente de redorer son blason et de faire valoir son architecture et ses espaces paysagers, même si, en réalité, la majorité de son économie tourne autour du jeu - interdit à Hong-Kong - et de la prostitution.

\section{Conclusion}

Il est important de souligner l'apparition d'institutions urbaines successives à Macao, réglementant la ville et son image, car leurs traces demeurent visibles. Le réseau viaire a subsisté dans sa majorité. Le style d'architecture portugais participe au charme de la ville et amène chaque jour une foule de visiteurs à prendre des photos « exotiques » en Chine, tout comme les axes comme l'avenue Almeida Ribeiro ou les plantations d'arbres qui constituent désormais l'identité touristique de Macao. 
Si aujourd'hui, la ville se construit en hauteur et met au premier plan le jeu et les casinos, comme à Las Vegas, elle reste néanmoins reconnue pour son patrimoine architectural portugais classé par l'UNESCO, comme dans l'avenue Almeida Ribeiro ou près du quartier da Praia, mais aussi certains parcs et les grottes de Luis de Camões construites en 1880. L'incidence de ces planifications urbaines sur la ville contemporaines se constate sur les

bâtiments historiques conservés et protégés. La trame urbaine historique est même reprise jusque dans les nouvelles avenues construites sur les terre-pleins pour y accueillir

les casinos, à l'instar de l'avenue do Infante Dom Henrique, véritable prolongement de l'avenue Almeida Ribeiro. La pensée urbaine de Macao est à l'origine de la ville que nous connaissons aujourd'hui.

\section{BIBLIOGRAPHIE}

ABREU ARRIMAR (DE), Jorge, « Sociedade e poder político em Macau nos séculos XVIII e XIX », Revista de Cultura de Macau, $\mathrm{n}^{\circ}$ 25, edição international, 2008, p.50-61

AGULHON Maurice,Histoire de la France Urbaine : la ville de l'âge industriel - le Cycle Haussmannien , T. 4, Paris, Le Point, 1998

AMARO Ana Maria,Das Cabanas de palhas as torres de betão

, Lisbonne, Universidade técnica de Lisboa, 1998

AZENHA CAÇAO Armando, « Sankiu »,Revista de Cultura de Macau

, n 35/36, Série II, 1998, p.132-147

CAÇORINO DA PALMA BARACHO Carlos Alberto

, Um percurso ao encontro do medieval em Macau: urbanismo e arquitectura: persistência e coexistência de elementos medievais portugueses e tradicionais chineses na estrutura urbana e arquitectónica de Macau nos séculos XVI e XVII

, Lisbonne, Université Nouvelle de Lisbonne, 1992

CALADO Maria, MENDES Maria Clara, TOUSSANT Michel, « Da Fundaçõ aos anos 70: Evolução sócio-económica, urbana e arquitectónica »,

Revista de Cultura de Macau,n³4, Série II, 1998, p.75-146

CONCEIÇÃo AFONSO (DA), José, « A Revolução Verde de Macau (século XIX - década 80) », Revista de Cultura de Macau, n³5/36, Série II, 1998, p.171-205

CONCEIÇÃO AFONSO (DA), José, « Macau: uma experiência de urbanismo estratégico e higienista dos finais do século XIX aos começos do século XX », Revista de Cultura de Macau, Série II, n³8/39, 1998, p.221-248

DIAS Pedro,A Urbanisação e a arquitectura dos portugueses em Macau: 1557 - 1911, Portugal Telecom Lisbonne, Lisbonne, 2005

DIAZ DE SEABRA Leonore, ESPADINHA Maria Antónia, « Para uma história das questões de saúde em Macau no século XIX: doenças, epidemias, médicos e doentes ", Revista de Cultura de Macau, $\mathrm{n}^{\circ}$ 25, edição international, 2008, p.50-61 
GRAÇA Jorge, « Urbanização de Macau e o mapa de Demétrio Démetrio Cinatti (1881) », Revista de Cultura de Macau, n³5/36, Série II, 1998, p.148-170

JORGE DA COSTA Monica,

L'urbanisation à Macao : entre traditions et modernité. D'un renouveau urbain à la ville d'aujourd'hui , Paris, mémoire de fin d'étude à l'Université Paris-I Panthéon-Sorbonne, 2014

PROENÇA DOS SANTOS BARREIROS Sergío,

Urbanismo colonial nas províncias orientais: continuidade e ruptura dos planos urbanísticos no Estado da India, Macau e Tímor, 1934 - 1974

, Lisbonne, Université technique de Lisbonne, 2007

RODRIGUES COST, Maria de Lourdes,História da Arquitectura em Macau,Macao,

Instituto Cultural, 1997

VIDEIRA PIRES Benjamin, « Os Governadores e a vida de Macau no Século XIX », Revista de Cultura de Macau, $\mathrm{n}^{\circ} 7$ et 8, 1988-1989, p.35-40

SIT, Victor F.SMacau ao longo de 500 anos, como surgiu e evoluiu uma cidade chinesa atípica , Hong Kong, Silkroad Press, 2013

\section{NOTES}

1.

AGULHON, Maurice, Histoire de la France urbaine : la ville de l'âge industriel. Le Cycle Haussmannien, T. 4, Paris, Le Point, 1998, p.12.

2.

Cet article est la synthèse de notre mémoire «L'urbanisation à Macao: entre traditions et modernité: D'un renouveau urbain à la ville d'aujourd'hui », présenté en 2014 dans le cadre du Master Erasmus Mundus TPTI, après le dépouillement des archives correspondantes à Macao.

3.

CAÇORINO DA PALMA BARACHO Carlos Alberto, Um percurso ao encontro do medieval em

Macau: urbanismo e arquitectura: persistência e coexistência de elementos medievais portugueses e tradicionais chineses na estrutura urbana e arquitectónica de Macau nos séculos XVI e XVII, Lisbonne, Université Nouvelle de Lisbonne, 1992.

4.

Cette notion de «technicien » regroupe ici ceux des savants, des médecins et des hommes politiques de Macao qui oeuvrèrent à repenser la ville.

5.

Le terme de Région Spéciale Administrée désigne les villes de Macao et Hong-Kong qui, par leur intégration récente, bénéficient aujourd'hui d'un statut spécial vis-à-vis du reste de la Chine (passeport Hong-kongais et Macanéen, législation différente, ouverture sur l'occident et présence des systèmes de communication interdits en Chine...).

6.

Nom utilisé par les habitants des Régions Spéciales Administrées pour parler du reste de la Chine. 7.

CONCEIÇÃO AFONSO (DA) José, « Macau: uma experiência de urbanismo estratégico e higienista dos finais do século XIX aos começos do século XX », Revista de Cultura de Macau, Série II, $\mathrm{n}^{\circ}$

$38 / 39,1998$, p.230.

8. CONCEIÇÃO AFONSO (DA), 1998, op. cit, p. 230. 
9.

GRAÇA Jorge, « Urbanização de Macau e o mapa de Demétrio Cinatti (1881) », Revista de Cultura de Macau, n³5/36, Série II, 1998, p.150.

10.

Les travaux des ports de Macao se succèderont durant près d'un demi-siècle et s'accompagneront de désenvasements ainsi que de la mise en place de terre-pleins. PROENÇA DOS SANTOS

BARREIROS Sergío, Urbanismo colonial nas províncias orientais: continuidade e ruptura dos planos urbanísticos no Estado da India, Macau e Tímor, 1934-1974, Lisbonne, Université technique de Lisbonne, 2007, p.101-105.

11.

C'est ainsi que Manuel de Castro Sampaio dénommait les habitants du Bazar Chinois, dans le centre ville de Macao.

12.

DE CASTRO SAMPAIO Manuel, os Chins de Macau, Hong Kong, Typographia de Noronha e filhos, 1867, p. 44-45. Traduit par mes soins.

e.

DIAZ DE SEABRA Leonore, ESPADINHA Maria Antónia, « Para uma história das questões de saúde em Macau no século XIX: doenças, epidemias, médicos e doentes », Revista de Cultura de Macau, $n^{\circ} 25$, edição international, 2008, p.50.

13.

Construites en bois, les maisons chinoises étaient peu adaptées pour faire face aux risques d'incendies, de typhons et aux insectes qui les rongeaient, CONCEIÇão AFONSO (DA) José, « A Revolução Verde de Macau (século XIX - década 80) », Revista de Cultura de Macau, n³5/36, Série II, 1998, p.176.

14.

Décret du 21 octobre 1863, cité dans CONCEIÇÃo AFONSO (DA) José, « Macau: uma experiência de urbanismo estratégico e higienista dos finais do século XIX aos começos do século XX », Revista de Cultura de Macau, Série II, n³8/39, 1998, p.226.

15. CONCEIÇÃO AFONSO (DA) José, ibidem, p.230.

16. Pour plus de détail sur le paysagisme et les politiques vertes de Macao au XIX siècle, voir l'article de CONCEIÇÃO AFONSO (DA) José, « A Revolução Verde de Macau (século XIX - década 80)», 1998, op.cit.

17.

AZENHA CAÇAO Armando, « Sankiu », Revista de Cultura de Macau, n 35/36, Série II, 1998, p.132-147.

18. CONCEIÇÃO AFONSO (DA) José, « Macau: uma experiência de urbanismo..., » op.cit.p. 232.

19. CONCEIÇÃO AFONSO (DA) José, «A Revolução Verde de Macau... », ibid. 


\section{RÉSUMÉS}

Comment penser la ville? Comment nos ancêtres ont-ils réfléchi au futur de leurs villes ? Cet article traite de l'apparition d'une pensée urbaine chez une élite administrative de Macao au XIXe siècle. Il en résultera un changement profond dans le paysage urbain. La ville construite spontanément avec ses quartiers désorganisés et parfois même insalubres, devint l'objet d'une vaste réflexion pour en faire une vitrine du futur, lui donner le visage neuf d'une ville en profonde mutation économique. L'étude de la fin du XIX

eet du début du XX

siècle nous mène à nous interroger sur l'évolution de la pensée urbaine, les nouveaux objectifs et les moyens mis en place par l'administration pour anticiper sur le futur de la ville. La trame urbaine de Macao en porte encore les traces.

How to think the city? How did our ancestors think their city of tomorrow ? This article deals with the appearance of an urban thinking amongst the adminsitrative elite of Macao, which will be at the origine of deep changes in the urban landscape of the city. The point is the evolution of

a spontaneously built city, with its desorganised and sometimes even unhealthy districts, to a more large-scaled thought about the city of tomorrow, a showcase for a new face of a city in deep economic change. The study of the end of the nineteenth century and the beginning of the twentieth century raises questions about the evolution of the urban thinking, the methods introduced and the new objectives of the administration regarding the future of the city, which nowadays is still visible in the urban fabric of Macao.

\section{INDEX}

Keywords : history of technology, contemporary history, Macao, urban thinking, XIXth century Mots-clés : Histoire des techniques, histoire contemporaine, Macao, pensée urbaine, XIXe siècle

\section{AUTEUR}

\section{MONICA JORGE DA COSTA}

Master II Techniques, Patrimoine et Territoires de l'Industrie Universités Paris I PanthéonSorbonne, Padoue (Italie) et Èvora (Portugal) 